Research Article

\title{
Diversity of the genus Agrilus Curtis (Coleoptera: Buprestidae) in South America
}

Diversidad del género Agrilus Curtis (Coleoptera: Buprestidae) en América del Sur

\author{
Gianfranco Curletti
}

${ }^{1}$ clo Museo Civico di Storia Naturale di Carmagnola, Parco Cascina Vigna, Carmagnola, Italy. E-mail: giancurletti@gmail.com

\section{ZooBank: urn:lsid:zoobank.org:pub: 9A6C7EA4-047C-4D01-A90F-89AEE5EB82DA https: / / doi.org/10.35249/ rche.46.3.20.09}

\begin{abstract}
Using an updated checklist of the Agrilus species of South America, it is analyzed the status of knowledge of the Agrilus fauna for each country on the continent. When the geographical extent of each country and the number of known species are considered, a great disparity of knowledge emerges, with some countries (e.g., French Guyana) being relatively well investigated and others (e.g., Ecuador, Peru, Colombia) for which the state of knowledge is still embryonic. It is therefore reasonable to expect the number of known species will increase greatly as further research is conducted, especially in the latter countries.
\end{abstract}

Key words: Agrilini, checklist, countries, density, distribution, Neotropics.

Resumen. A partir de una lista actualizada de las especies de Agrilus de América del Sur, se propone un análisis del estado del conocimiento de la fauna de Agrilus para cada país del continente. Considerando la extensión de los diferentes países y el número de especies descubiertas hasta ahora, surge una gran disparidad de conocimiento, con países relativamente bien investigados (e.g., Guyana Francesa) en comparación con otros para los cuales el estado del conocimiento es incipiente (e.g., Ecuador, Perú, Colombia). Por lo tanto, es razonable esperar un gran aumento futuro del numero de especies conocidas a partir de nuevas investigaciones.

Palabras clave: Agrilini, listado, países, densidad, distribución, Neotrópico.

\section{Introduction}

The genus Agrilus Curtis, 1825 is one of the most species-rich genera in the entire animal kingdom and occurs on all the continents around the world except Antarctica. Based on data gathered over many years of research and with the help of data kindly provided to me by esteemed colleagues Henry Hespenheide and Eduard Jendek, the number of taxa currently known is estimated at just over 4,000 (Table 1, Fig. 1), number surely conservative. What is most surprising in the study of this vast genus is that its taxonomic knowledge and its numerical consistency are still far from exhausted. In fact, any field research carried out in tropical or subtropical areas or even simply the study of historical museum collections inevitably leads to the discovery of many undescribed species.

Statistical projections conducted on limited regions of the world (Curletti 2006;

Received 13 June 2020 / Accepted 28 July 2020 / Published online 28 August 2020

Responsible Editor: José Mondaca E. 
Hespenheide 1996; Hespenheide \& Chaboo 2015) have hypothesized a significant number of species that are still unknown, up to double or even quadruple the number of currently known species. It follows from the evidence that certain regions of the world are still poorly explored, both in taxonomic and numerical terms.

The intent of this paper is to attempt to establish the state of knowledge of the Agrilus fauna in each country of South America and to establish a relationship between the number of taxa present and its geographic extent. To obtain this result, we considered it useful to list all reported taxa.

Table 1. Approximate number of Agrilus taxa in the world.

\begin{tabular}{|c|c|}
\hline Countries & Taxa \\
\hline Palearctic & 490 \\
\hline Ethiopian & 945 \\
\hline Nearctic & 200 \\
\hline Neotropical & 1430 \\
\hline Oriental & 880 \\
\hline Australian & 100 \\
\hline Total & 4045 \\
\hline
\end{tabular}

Methods and Considerations. Fundamental importance in this study is the Bellamy catalog (2008) which is comprehensive until the year of its publication, both as regards taxa and bibliography. For this reason, the bibliography has been updated by omitting the previous contributions and limited to adding the later ones. In addition to this, the data presented in the following tables are based on the knowledge acquired by years of studies of the taxa in each country. The main purpose is to highlight the current state of knowledge and the degree of knowledge of the Agrilus fauna based on the relationship between the number of species and the geographical extent of the country. The absolute numbers for individual countries (Table 2, Fig. 2) can lead to erroneous or meaningless conclusions, as they are subject to numerous variables such as climate, vegetational characteristics, difficulty of access during historical periods, and orography.

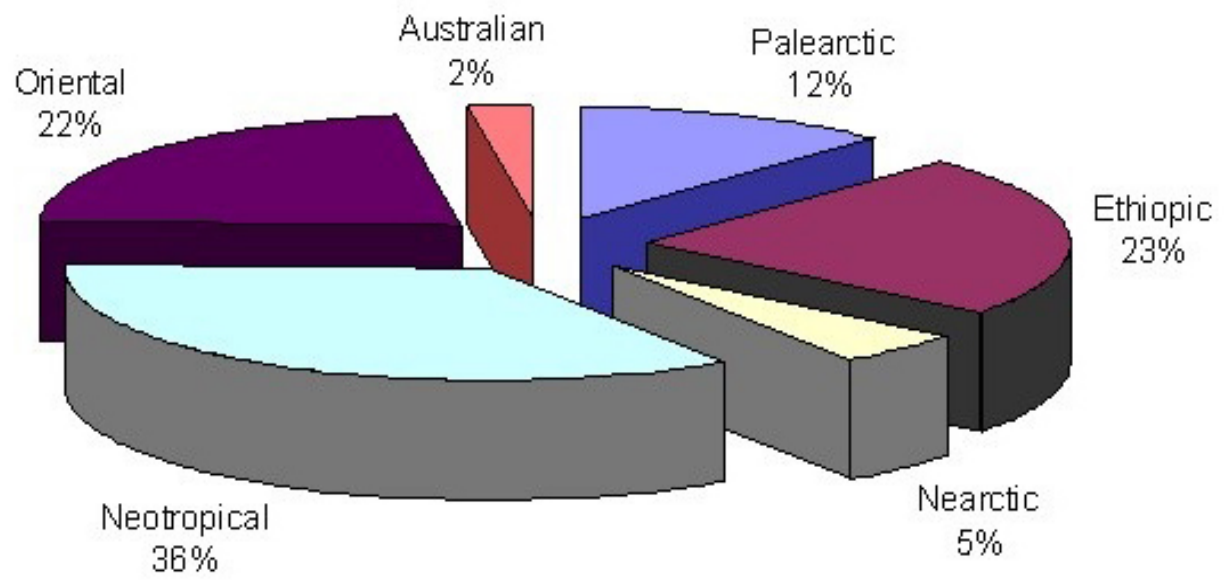

Figure 1. Percentage subdivision of taxa in the Zoogeographic Regions. Neotropics is the richest in species. 
Table 2. Column 1 lists the countries of South America, column 2 gives the surface area of each country in $\mathrm{km}^{2}$, column 3 gives the total number of described taxa, column 4 gives the number of synonymized or excluded taxa, and column 5 gives the net number of taxa after excluding the number in column 4 .

\begin{tabular}{|l|c|c|c|c|}
\hline Country & \multicolumn{1}{|l|}{$\mathbf{k m}^{\mathbf{2}}$} & spp. & syn. & Tot. \\
\hline Trinidad \& Tobago & 4768 & 13 & 0 & 13 \\
\hline French Guyana & 83534 & 155 & 7 & 148 \\
\hline Colombia & 1142000 & 34 & 5 & 29 \\
\hline Venezuela & 916445 & 26 & 1 & 25 \\
\hline Ecuador & 283560 & 9 & 0 & 9 \\
\hline Guyana & 214969 & 11 & 5 & 6 \\
\hline Suriname & 163821 & 0 & 0 & 0 \\
\hline Brazil & 8511000 & 550 & 57 & 493 \\
\hline Peru & 1285000 & 34 & 0 & 34 \\
\hline Bolivia & 1099000 & 24 & 2 & 22 \\
\hline Chile & 756950 & 10 & 5 & 5 \\
\hline Paraguay & 406752 & 27 & 4 & 23 \\
\hline Argentina & 2780000 & 136 & 29 & 107 \\
\hline Uruguay & 176215 & 14 & 3 & 11 \\
\hline
\end{tabular}

Orography and latitude. Have fundamental importance, allowing to distinguish tropical and subtropical areas, summarily treated in the discussion.

Vegetation. In particular the presence of forests, is of great importance, but as far as Neotropical Agrilus are concerned, many species have evolved away from woody plants and adapted to herbaceous plants-much more so than the African, Palearctic and Australian faunas. Such species are particularly present in savannah (Brazilian Cerrado, Argentine Pampas) or sub-desert areas (Chile and Peru). Hespenheide (1991) noted that leaf-miners (Tracheini) are predominant in southern Neotropical habitats. There are no known Agrilus leaf miners; however, an ecological convergence can be seen by the considerable number of stem-boring species that attack living plants, both herbaceous (Fig. 5) and woody. This obviously implies a high percentage of host specificity that, at least in part, explains the difficulty in encountering individuals of these species, in contrast to what occurs in the faunas of other geographic areas. Access to forests has also been a significant factor in faunistic explorations during historical times. For example, during the 18th and 19th centuries, the Brazilian Amazon rainforest remained almost completely inaccessible for research, contrasting sharply with the Atlantic forest. Accordingly, a significant percentage of species described in Brazil $(71 \%)$ are found in forests along the Atlantic Ocean, while only $6 \%$ are from Amazonian forests (Curletti 2018a, 2019).

Territorial dimension. Is the only variable that can be precisely quantified, and it is on this metric that the considerations presented here are based, albeit empirically. While imperfect, it does provide some useful indications on the state of knowledge of the Agrilus fauna. 


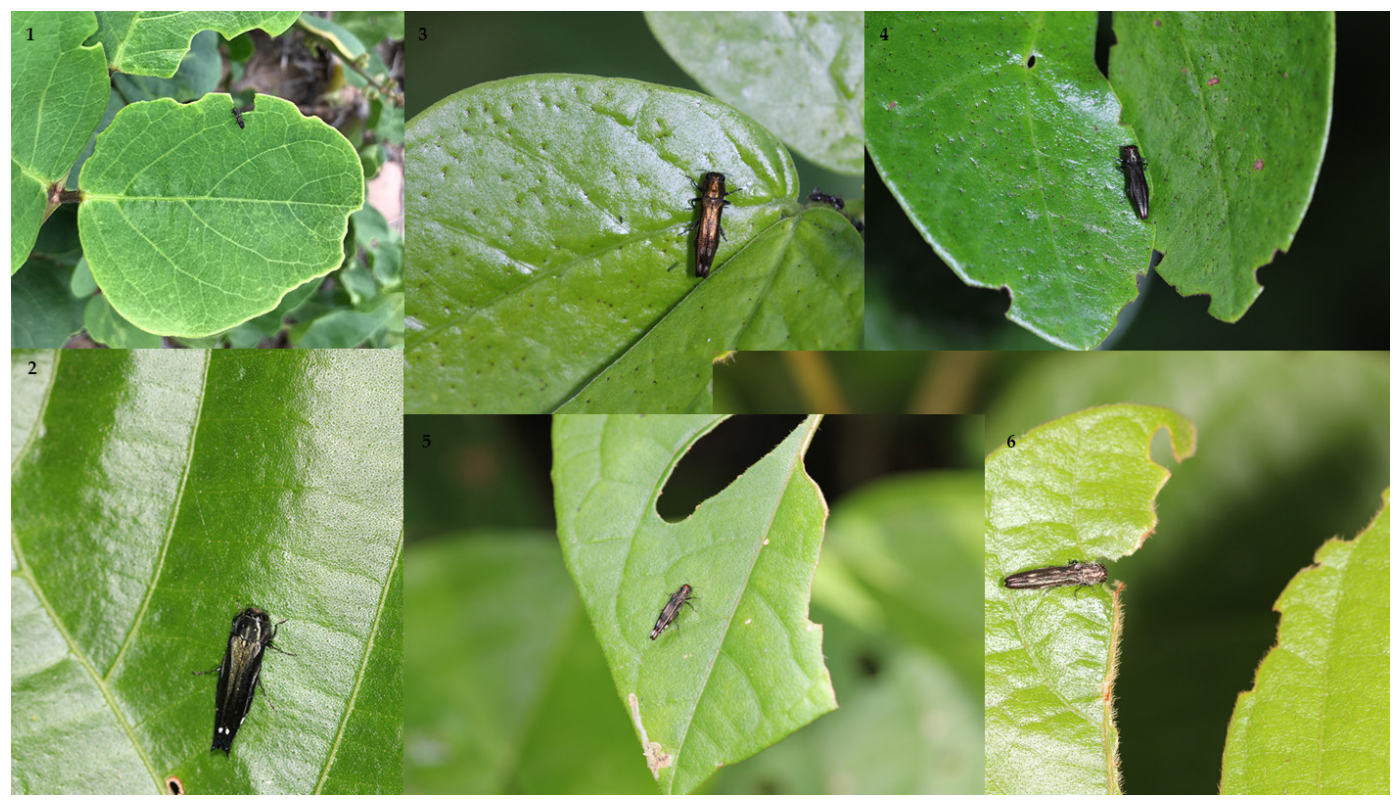

Figure 5. 1. Agrilus sp. cfr. figuratus Curletti \& Brûlé on a rear dune herbaceous plant. Brazil, Bahia (photo F. Albertoni). 2. Agrilus piscis Gory on Senna quinquangulata. French Guyane, Route de Kaw, PK 37, sur Senna quinquangulata (photo S. Brûlé). 3. Agrilus lopus Obenberger on Senna cfr. chrysocarpa. French Guyane, Rés. des Nouragues (photo S. Brûlé). 4. Agrilus intermixtus Curletti \& Brûlé on Senna cfr. chrysocarpa. French Guyane, Rés. des Nouragues (photo S. Brûlé). 5. Agrilus goryellus Thomson. French Guyane, Route de Kaw, on Mendoncia hoffmannseggiana (photo S. Brûlé). 6. Agrilus trames Curletti \& Brûlé. French Guyane, Rémire-Montjoly, on Mucuna sloanei (photo S. Brûlé).

\section{Observations and Conclusions}

As can be seen in Table 2 (column 5) and Fig. 2, absolute numbers of taxa suggest that Brazil is the best-investigated country regarding its Agrilus fauna, followed by Peru and Argentina. The absolute numbers also suggest that countries with smaller geographical extents and, thus, faunistically poorer such as Trinidad and Tobago, Ecuador, or Paraguay, are less well investigated.

By examining the relationship between known species and geographical extent (Fig. 3), the percentages highlight how French Guyana and Trinidad and Tobagothe latter especially despite fewer total taxa-are notably richer in species and, thus, the best-known faunistically, as confirmed by the contributions of Fisher $(1943,1944)$ for Trinidad and Tobago and Curletti \& Brûlé (2011, 2013, 2014a, 2014b, 2014c, 2015, 2016, 2017, 2018) for French Guyana. In contrast, Brazil, despite more than 540 known species, has not been as well explored faunistically compared to the previous two or Uruguay. For the remaining countries, there are no reports from Suriname and relatively few from Bolivia and Colombia relative to their geographical extent.

These results suggest that nations such as Venezuela, Peru, and Ecuador deserve greater investigation, since they are poorly explored relative to their geographical extent.

Chile represents an exception due to the objective scarcity of species resulting from its relatively cold, desert climate. The data may also be less objective for Argentina (Curletti 2018), since only the northern part of the country has a subtropical climate and the southern part partially shares the Chilean climate, although less so in the eastern part due to the greater distance from the Andes Mountains. The remaining 
countries intersected by the Andes Mountains (Peru, Colombia, Ecuador) all have warm-climate rainforests.

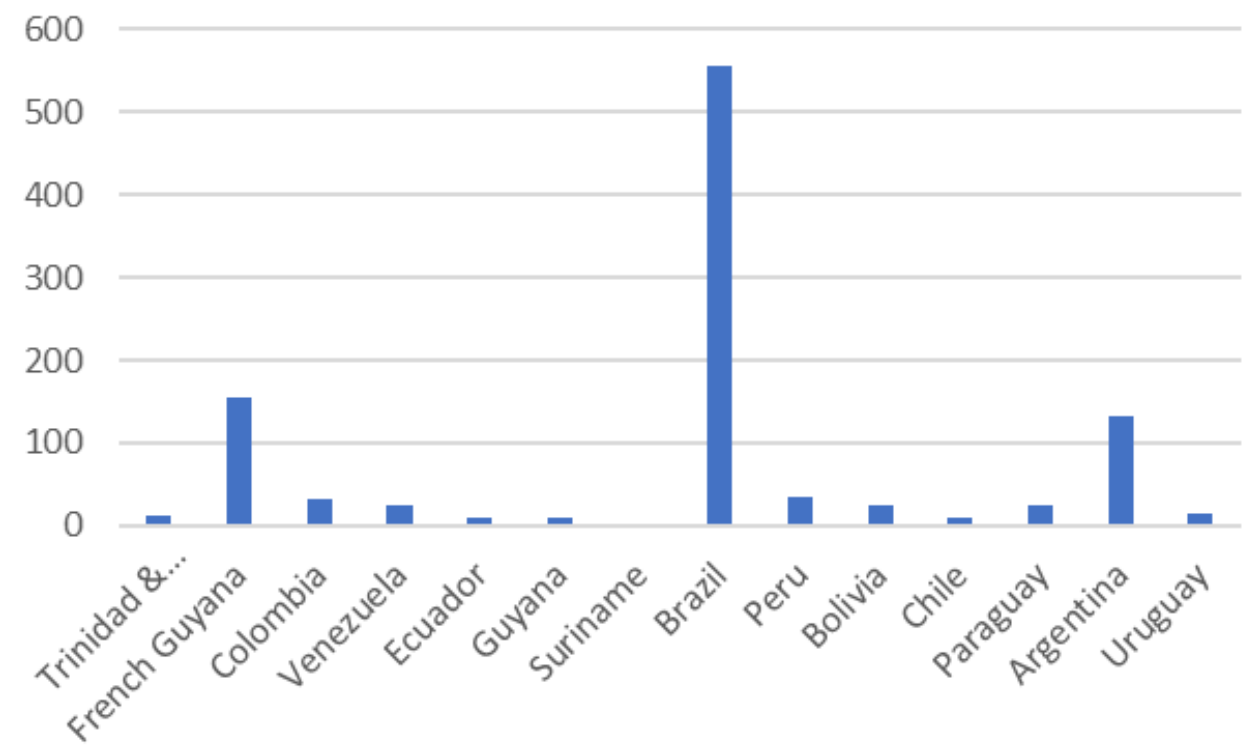

Figure 2. Number taxa/ country for South America.

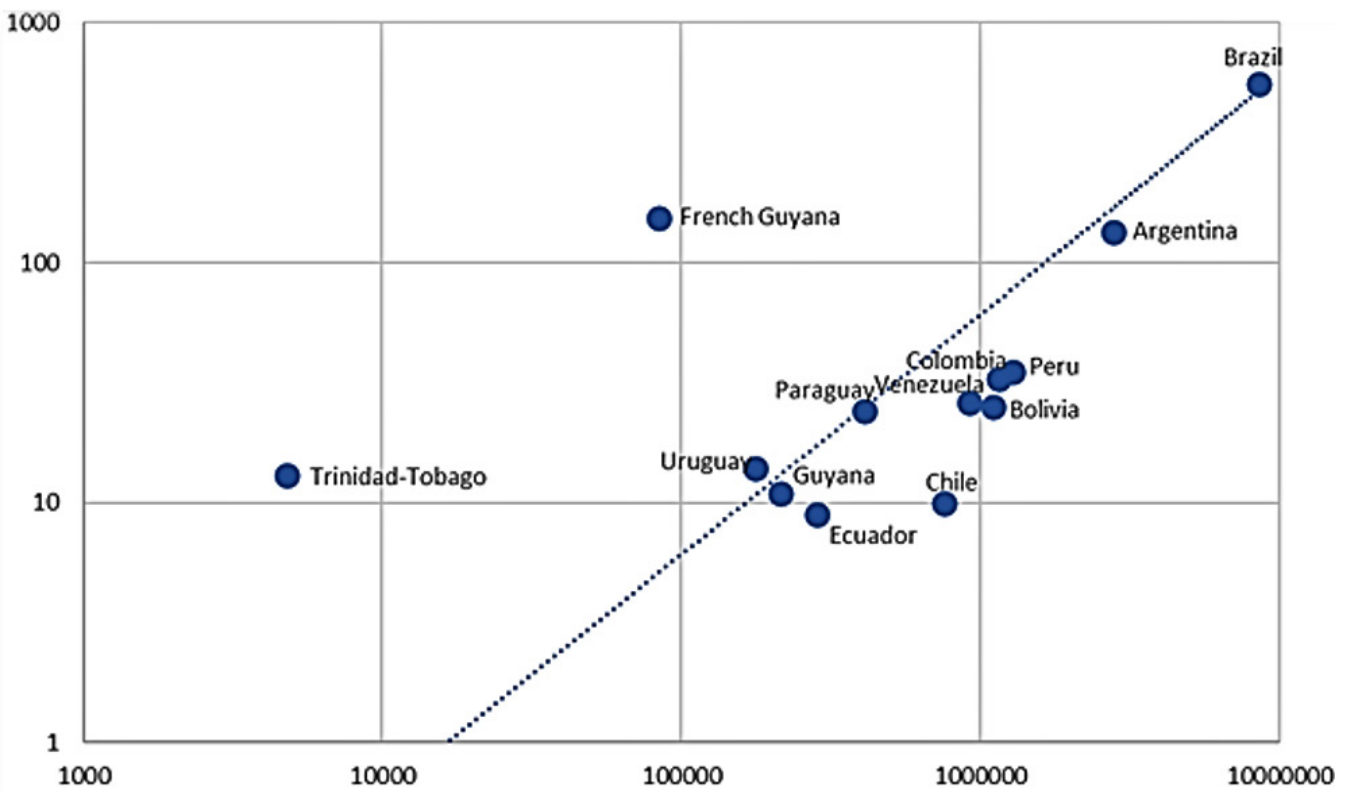

Figure 3. Species / area curve for Agrilus spp. in South America: number of Agrilus spp. of countries in South America in relation to their geographical area. The curve is highly likely skewed based on the state of knowledge of the entomofauna of each country and the number of species in countries such as Venezuela, Colombia, Peru and Ecuador will surely increase greatly with further research. Number of species is also greatly influenced by ecological and biogeographical factors such as climate, topography, geographical barriers (mountains and rivers), etc. Looking at the graph, however, it is clear that French Guyana and Trinidad Tobago are differentiated from the average. Brazil has the highest number of species but falls within the average knowledge of other states. On the opposite side is Chile for the reasons explained in the text. 
Regarding the study of the genus Agrilus in South America (Fig. 4), the greatest interest has occurred from the end of the 19th century through the first half of the 20th century. Most of the progress during these years was due primarily to two authors: Charles Kerremans during the late 1800s and Jan Obenberger during the early 1900s, who have been able to take advantage of research carried out by talented researchers such as Dirings, Mraz, Pereira, Plaumann in Brazil, Peña in Chile. Subsequent to this period there was a sharp decline in research until the present day, which has seen a marked recovery due primarily to the contributions of Curletti and several collaborators, thanks mainly to the collections made by SEAG members in French Guyana and the study of material preserved in the museums of Berlin, Ilha Solteira, Lima, Manaus, Ouro Preto, São Paulo. In addition to these, marginal contributions for taxonomic corrections (Bellamy 1998; Jendek 2001, 2005, 2012).

The enormous amount of material that is awaiting study remains in the European and North American museums, not to mention the university entomological collections scattered in hundreds in the South American countries.

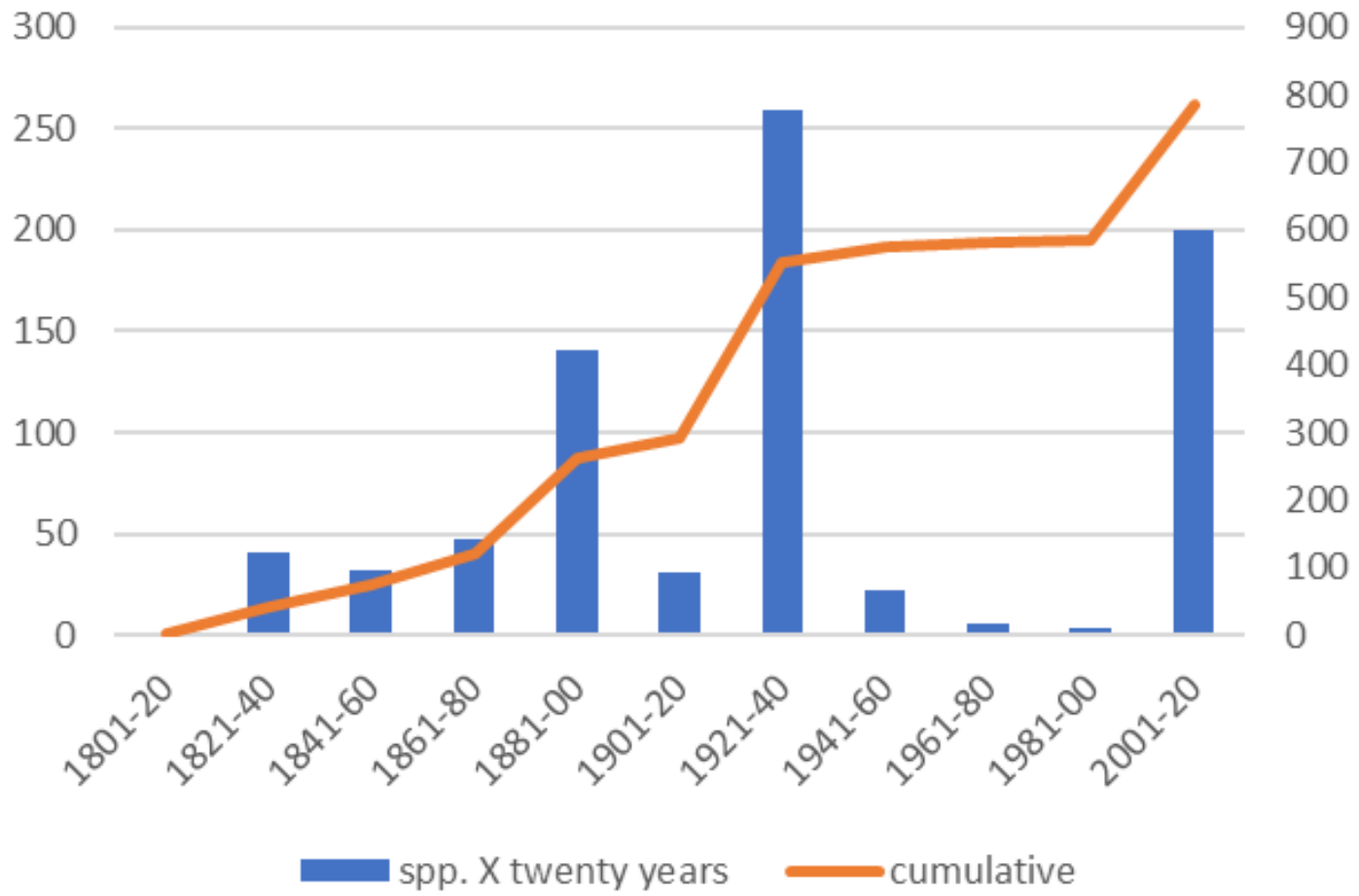

Figure 4. Trend related to the progress on the study of the genus Agrilus over the past twenty years. It is evident that the highest activity occurred in the early 1900s due to the contributions of Kerremans and Obenberger. There is a substantial drop in activity afterwards before a renewal of research activity beginning in 2000 .

\section{Acknowledgments}

I would like to thank colleagues Giovanni Boano, Stéphane Brûlé, Angelo Dutto, Henry Hespenheide and Eduard Jendek for their suggestions, help, collaboration and to Ted MacRae, Letizia Migliore and Cristian Pineda for linguistic help. 
Appendix 1. List of known taxa for each country. Generic records such as "South America”, "Tropical America" or "Agrilus sp." were not considered in this list.

\section{Argentina}

Agrilus aenescens Kerremans, 1903

Agrilus aeneus Gory \& Laporte, 1837

Agrilus aesquilius Obenberger, 1947

Agrilus agrius Obenberger, 1935

Agrilus albomaculifer albomaculifer Saunders, 1870

Agrilus albomaculifer circumflutus Obenberger, 1947

Agrilus alcinous Obenberger, 1935

Agrilus alexandri Obenberger, 1933

Agrilus angustus (Chevrolat, 1835)

Agrilus antonii Obenberger, 1933

Agrilus argentinus Kerremans, 1903

Agrilus aristaeus Kerremans, 1913

Agrilus arnus Gory, 1841

Agrilus aurelianus Obenberger, 1947

Agrilus auriginus Curletti, 2018a

Agrilus aurocephalus Gory, 1841

Agrilus bellicus Kerremans, 1903

Agrilus bergi Kerremans, 1903

Agrilus blanchardii Saunders, 1870

Agrilus bonariensis Kerremans, 1903

Agrilus bordoni Curletti, 2018a

Agrilus bruchi Kerremans, 1903

Agrilus bruchianus Obenberger, 1933

Agrilus carinus carinus Obenberger, 1935

Agrilus carinus matutinus Obenberger, 1935

Agrilus caroli Kerremans, 1903

Agrilus casignetus Obenberger, 1932

Agrilus catamarcanus Obenberger, 1916

Agrilus cernosvitovi Obenberger, 1935

Agrilus chacoensis Obenberger, 1935

Agrilus chrysostictus chrysostictus (Klug, 1825)

Agrilus clairvillei Obenberger, 1933

Agrilus desfontainesi Obenberger, 1933

Agrilus dilaticornis Kerremans, 1897

Agrilus erubescens Curletti, 2018a

Agrilus eurysaces Obenberger, 1947

Agrilus facetus Curletti, 2018a

Agrilus faciatus Kerremans, 1903

Agrilus filiolus Obenberger, 1933

Agrilus flaveolus Gory \& Laporte, 1837

Agrilus flosculus Obenberger, 1933

Agrilus friesei Obenberger, 1932

Agrilus frigidus Gory, 1841

Agrilus fulvolineatus Kerremans, 1903

Agrilus furcatipennis Gory \& Laporte, 1837

Agrilus graciae Obenberger, 1932

Agrilus granulicollis Gory \& Laporte, 1837 
Agrilus guarani Obenberger, 1933

Agrilus guardianus Curletti, 2020a

Agrilus gumia Obenberger, 1935

Agrilus honorius loretigena Obenberger, 1933

Agrilus idas Obenberger, 1935

Agrilus ignavus Kerremans, 1897

Agrilus inauratum Curletti, 2018a

Agrilus japyx Obenberger, 1935

Agrilus jarbas Obenberger, 1935

Agrilus kaszabi Pochon, 1967

Agrilus llanero Obenberger, 1933

Agrilus loretanus Obenberger, 1933

Agrilus maculisternis Obenberger, 1932

Agrilus mandatus Kerremans, 1913

Agrilus meleager meleager Obenberger, 1935

Agrilus miron Obenberger, 1933

Agrilus missionum Obenberger, 1947

Agrilus multispinosus (Klug, 1825)

Agrilus neuquensis Kerremans, 1903

Agrilus niger Gory \& Laporte, 1837

Agrilus nivatus Curletti, 2020a

Agrilus nobilis Burmeister, 1872

Agrilus noviusculus Curletti, 2018a

Agrilus nudidorsis Curletti, 2015b

Agrilus obscuripennis Gory, 1841

Agrilus omissus Kerremans, 1903

Agrilus onca Curletti, 2020a

Agrilus pauperculus Gory, 1841

Agrilus perplexus Burmeister, 1872

Agrilus piliferus Kerremans, 1897

Agrilus praecipuus Kerremans, 1897

Agrilus puellus Gory \& Laporte, 1837

Agrilus quilmesinus Obenberger, 1933

Agrilus reimoseri Obenberger, 1933

Agrilus rodzjankoi Obenberger, 1933

Agrilus rugosicollis Blanchard, 1846

Agrilus sacer ssp. bomplandellus Obenberger, 1933

Agrilus sacer ssp. tremolerasi Obenberger, 1933

Agrilus saltensis Kerremans, 1903

Agrilus schreineri Obenberger, 1933

Agrilus senior Curletti, 2018a

Agrilus spinamajor Chevrolat, 1838

Agrilus strephon Obenberger, 1933

Agrilus subinflatus Kerremans, 1899

Agrilus sulcipennis Solier, 1849

Agrilus sulphurifer Burmeister, 1872

Agrilus thianshanskii Obenberger, 1933

Agrilus thoracellus ssp. thoracellus Gory \& Laporte, 1837

Agrilus thoracicus Gory \& Laporte, 1837

Agrilus tractatus Curletti, 2018a

Agrilus triseriatus Curletti, 2018a

432 
Agrilus tucumanellus Obenberger, 1933

Agrilus tucumanus Kerremans, 1903

Agrilus venustulus Burmeister, 1872

Agrilus verutus Kerremans, 1897

Agrilus vezenyii Pochon, 1967

Agrilus vianai Obenberger, 1947

Agrilus wagneri Kerremans, 1913

Agrilus wasmanni ssp. yerbatero Obenberger, 1933

Agrilus xylochaerus Obenberger, 1947

\section{Bolivia}

Agrilus altitudinalis Curletti, 2015b

Agrilus auritus Chevrolat, 1838

Agrilus beniensis Fisher, 1925

Agrilus boliviensis Fisher, 1925

Agrilus buenavistae Obenberger, 1933

Agrilus cavinas Fisher, 1925

Agrilus celsus Kerremans, 1897

Agrilus chaparensis Pochon, 1971

Agrilus curvicollis Moore, 1986

Agrilus dorbignyi Saunders, 1870

Agrilus eupalamus Gory, 1841

Agrilus gorai Fisher, 1925

Agrilus honorius Obenberger, 1933

Agrilus koshantshikovi Obenberger, 1935

Agrilus manni Fisher, 1925

Agrilus saussurei Obenberger, 1933

Agrilus strombus Obenberger, 1933

Agrilus strophius Obenberger, 1933

Agrilus takana Fisher, 1925

Agrilus tumultuosus Obenberger, 1932

Agrilus tumupasaensis Fisher, 1925

Agrilus violaceicollis Gory, 1841

\section{Brazil}

Agrilus aculeatus Gory, 1841

Agrilus aculeipennis Chevrolat, 1838

Agrilus adamanteus Curletti \& Pineda, 2018

Agrilus adansoni Obenberger, 1933

Agrilus adelungi Obenberger, 1933

Agrilus adustellus Thomson, 1879

Agrilus aebii Pochon, 1971

Agrilus aegrotus Curletti \& Migliore, 2014b

Agrilus aeneellus Thomson, 1879

Agrilus aeneifrons Kerremans, 1903

Agrilus aenescens Kerremans, 1903

Agrilus aeneus Gory \& Laporte, 1837

Agrilus aestimatus Kerremans, 1897

Agrilus affabilis Kerremans, 1897

Agrilus agronomus Obenberger, 1935

Agrilus albomaculifer Saunders, 1870 
Agrilus albonotatus Thomson, 1879 Agrilus albovittatus Thomson, 1879 Agrilus alini Curletti, 2016 Agrilus allectorellus Thomson, 1879 Agrilus almenus Gory, 1841 Agrilus amazo Obenberger, 1933 Agrilus amazonicus Kerremans, 1897 Agrilus amoenulus Kerremans, 1903 Agrilus analis Kerremans, 1896 Agrilus anceps Curletti \& Migliore, 2014b Agrilus antepodex Thomson, 1879 Agrilus apellos Pochon, 1971 Agrilus aragonis Obenberger, 1933 Agrilus araneipes Obenberger, 1932 Agrilus ardosiellus Obenberger, 1933 Agrilus armillatus Kerremans, 1903 Agrilus arnus Gory, 1841 Agrilus aurifrons Kerremans, 1897 Agrilus auritus Chevrolat, 1838 Agrilus aurocephalus Gory, 1841 Agrilus aurofissum Curletti, 2019b Agrilus auroscutatus Curletti \& Brûlé, 2011 Agrilus avulsus Kerremans, 1903 Agrilus azureus Kerremans, 1899 Agrilus badius Kerremans, 1897 Agrilus bahianus Curletti, 2020a Agrilus baliolus Kerremans, 1897 Agrilus banksi Obenberger, 1933 Agrilus barrosi Curletti \& Pineda, 2018 Agrilus bedelianus Obenberger, 1933 Agrilus bellator Kerremans, 1900 Agrilus bergrothi Obenberger, 1923 Agrilus biankii Obenberger, 1933 Agrilus bicarinatus Waterhouse, 1889 Agrilus bifenestratus Thomson, 1879 Agrilus bifenestrellatus, Obenberger, 1935 Agrilus bifoveicollis Kerremans, 1897 Agrilus bikini Curletti \& Brûlé, 2013b Agrilus bistrilineatus Obenberger, 1935 Agrilus blanditiosus Obenberger, 1933 Agrilus bondroiti Obenberger, 1933 Agrilus braemi Obenberger, 1933 Agrilus brasilius Bellamy, 1998 Agrilus bremus Obenberger, 1935 Agrilus bronzeellus Thomson, 1879 Agrilus buffoni Obenberger, 1933 Agrilus buyssoni Obenberger, 1933 Agrilus caesareus Obenberger, 1933 Agrilus caminosus Obenberger, 1933 Agrilus campesinus Curletti, 2019a Agrilus camusi Curletti \& Brûlé, 2011 
Agrilus capillitectus Obenberger, 1933

Agrilus cardiaspis Kerremans, 1897

Agrilus carinellus Thomson, 1879

Agrilus carinus ssp. carinus Obenberger, 1932

Agrilus cariosus Kerremans, 1899

Agrilus cartesias Obenberger, 1933

Agrilus cartistus Obenberger, 1932

Agrilus casarii Curletti \& Migliore, 2014b

Agrilus caudalis Gory \& Laporte, 1837

Agrilus caudatus Mannerheim, 1837

Agrilus celsus Kerremans, 1897

Agrilus chiarae Curletti, 2019b

Agrilus chrysostictoides brutus Obenberger, 1935

Agrilus chrysostictoides chrysostictoides Obenberger, 1935

Agrilus chrysostictus chrysostictus (Klug, 1825)

Agrilus chrysostictus comizon Obenberger, 1935

Agrilus cingulatus Kerremans, 1897

Agrilus circus Obenberger, 1932

Agrilus cisses Obenberger, 1932

Agrilus citrinolatus Thomson, 1879

Agrilus clangedon Obenberger, 1933

Agrilus clausus Kerremans, 1897

Agrilus clavaraui Obenberger, 1933

Agrilus clazon Obenberger, 1933

Agrilus cleidecostae Curletti, 2020a

Agrilus clown Curletti, Ribeiro \& Migliore, 2016

Agrilus coal Curletti, Ribeiro \& Migliore, 2013

Agrilus colleti Curletti \& Brûlé, 2011

Agrilus colluviellus Thomson, 1879

Agrilus comptus Kerremans, 1900

Agrilus comstocki Obenberger, 1933

Agrilus congener Kerremans, 1896

Agrilus conicus Gory, 1841

Agrilus consentaneus Kerremans, 1897

Agrilus consularis Kerremans, 1897

Agrilus contristatus Obenberger, 1935

Agrilus contrucidatus Obenberger, 1935

Agrilus copraeus Obenberger, 1932

Agrilus corysson Obenberger, 1933

Agrilus costifer Kerremans, 1903

Agrilus costipennis Kerremans, 1899

Agrilus crapulellus Thomson, 1879

Agrilus credulus Kerremans, 1897

Agrilus crucifer Curletti, 2020a

Agrilus crux Curletti \& Migliore, 2014b

Agrilus ctenocerus ctenocerus Gory, 1841

Agrilus ctenocerus mrazianus Obenberger, 1933

Agrilus cupido Obenberger, 1924

Agrilus cupidus Kerremans, 1897

Agrilus cuprescensellus Thomson, 1879

Agrilus curator Kerremans, 1903 
Agrilus cuvieri Obenberger, 1933

Agrilus cyanopterus Fabricius, 1801

Agrilus daubentoni Obenberger, 1933

Agrilus decandolli Obenberger, 1933

Agrilus decemnotatus Chevrolat, 1838

Agrilus deceptor Kerremans, 1897

Agrilus deceptorosus Obenberger,1933

Agrilus decorus Kerremans, 1897

Agrilus deliciosus Kerremans, 1897

Agrilus dentipennis Kerremans, 1903

Agrilus depressifrons Kerremans, 1900

Agrilus depressus Kerremans, 1899

Agrilus desfontainesi Obenberger, 1933

Agrilus desideratus Kerremans, 1897

Agrilus desmaresti Obenberger, 1932

Agrilus diagoros Obenberger, 1933

Agrilus diaphanes Obenberger, 1933

Agrilus dicalis Kerremans, 1903

Agrilus dicax Kerremans, 1897

Agrilus dichrous Kerremans, 1897

Agrilus differens Kerremans, 1896

Agrilus dilaticornis minarum Obenberger, 1933

Agrilus dilaticornis paulinus Obenberger, 1933

Agrilus dioscorides Obenberger, 1935

Agrilus dirus Kerremans, 1897

Agrilus disorientatus Curletti, Ribeiro \& Migliore, 2013

Agrilus dives Kerremans, 1897

Agrilus docilis Kerremans, 1897

Agrilus dolatus Kerremans, 1897

Agrilus dualis Kerremans, 1903

Agrilus dubiosus Curletti \& Migliore, 2014b

Agrilus dubius Kerremans, 1897

Agrilus duckei Curletti, 2019b

Agrilus dudai Obenberger, 1932

Agrilus duplicatus Kerremans, 1896

Agrilus dysauxes Obenberger, 1933

Agrilus elaphrus Obenberger, 1933

Agrilus electus Kerremans, 1897

Agrilus elimatus Obenberger, 1935

Agrilus enervatus Thomson, 1879

Agrilus epaulus Obenberger, 1932

Agrilus erbeni Obenberger, 1933

Agrilus espiritosus Curletti, 2020c

Agrilus eudaemon Obenberger, 1933

Agrilus eulaxus Obenberger, 1932

Agrilus eutyches Obenberger, 1932

Agrilus evansiae Obenberger, 1933

Agrilus eversor Kerremans, 1903

Agrilus exageratus Obenberger, 1933

Agrilus eximius Kerremans, 1897

Agrilus expletus Kerremans, 1897

436 
Agrilus expolitus Kerremans, 1897

Agrilus faber Kerremans, 1897

Agrilus fabricii Obenberger, 1993

Agrilus fasattii (Obenberger, 1943)

Agrilus fasciatellus Thomson, 1879

Agrilus fasciolatus Chevrolat, 1838

Agrilus fastidiosus Kerremans, 1896

Agrilus fastigatus Kerremans, 1897

Agrilus faurei Curletti \& Brûlé, 2011

Agrilus femina Curletti \& Migliore, 2014b

Agrilus fertoni Obenberger, 1933

Agrilus figuratus Curletti \& Brûlé, 2011

Agrilus filonius Obenberger, 1933

Agrilus filosellus Thomson, 1879

Agrilus flaveolus Gory \& Laporte, 1837

Agrilus fletchmanni Curletti \& Migliore, 2014a

Agrilus fontenellei Curletti, 2020c

Agrilus fragmentatus Curletti \& Brûlé, 2011

Agrilus fraudatorellus Thomson, 1879

Agrilus fulvolineatus Kerremans, 1903

Agrilus furcatipennis Gory \& Laporte, 1837

Agrilus furiosus Obenberger, 1935

Agrilus fusicauda Curletti \& Migliore, 2014b

Agrilus galileae Curletti \& Migliore, 2013

Agrilus garrulus Kerremans, 1903

Agrilus gaudens Kerremans, 1897

Agrilus gebhardti Obenberger, 1935

Agrilus geminus Curletti \& Migliore, 2014b

Agrilus generosus Kerremans, 1896

Agrilus geoffroyi Obenberger, 1933

Agrilus giannii Curletti \& Migliore, 2014b

Agrilus gigii Curletti, 2020a

Agrilus gileti Obenberger, 1933

Agrilus gilvopictus Kerremans, 1897

Agrilus globulus Curletti \& Migliore, 2014b

Agrilus goryellus Thomson, 1879

Agrilus goryi Saunders, 1870

Agrilus gounellei Kerremans, 1897

Agrilus goyaz Bellamy, 1998

Agrilus gracchus Obenberger, 1935

Agrilus granulicollis Gory \& Laporte, 1837

Agrilus gratus Kerremans, 1899

Agrilus gravenhorsti Obenberger, 1935

Agrilus grilloi Obenberger, 1933

Agrilus grouvellei Kerremans, 1896

Agrilus guttifer Kerremans, 1900

Agrilus gyllenhalius Obenberger, 1933

Agrilus haenkei Obenberger, 1932

Agrilus hansi Obenberger, 1933

Agrilus herbophagus Curletti, 2016

Agrilus herostratus Obenberger, 1935 
Agrilus hesperus Kerremans, 1897

Agrilus hexacanthus Obenberger, 1935

Agrilus hieroglyphicus Kerremans, 1899

Agrilus honestus Kerremans, 1896

Agrilus honorius honorius Obenberger, 1933

Agrilus honorius mineiranus Obenberger, 1933

Agrilus hoschekanus Obenberger, 1933

Agrilus humboldti Obenberger, 1933

Agrilus hylaeae Obenberger, 1933

Agrilus hypatus Obenberger, 1933

Agrilus hypochoreon Obenberger, 1933

Agrilus ichthyocerus Perty, 1830

Agrilus ignarus Kerremans, 1897

Agrilus ignavus Kerremans, 1897

Agrilus ignicaudatellus Thomson, 1879

Agrilus ignipennis Lucas, 1858

Agrilus imitator Obenberger, 1933

Agrilus immaculatellus Thomson, 1879

Agrilus immaculicollis Thomson, 1879

Agrilus immsi Obenberger, 1933

Agrilus impar Kerremans, 1897

Agrilus impressipennis Thomson, 1879

Agrilus impudens Kerremans, 1897

Agrilus inachus Obenberger, 1935

Agrilus indagator Kerremans, 1900

Agrilus indulgens Kerremans, 1897

Agrilus infelix Gory, 1841

Agrilus inflatus Kerremans, 1897

Agrilus infuscatus Kerremans, 1897

Agrilus inornatus Kerremans, 1896

Agrilus intermedius Kerremans, 1897

Agrilus irregularis Kerremans, 1899

Agrilus josei Obenberger, 1925

Agrilus julius Obenberger, 1933

Agrilus jussieui Obenberger, 1933

Agrilus kerremansellus Obenberger, 1935

Agrilus kirbyi Obenberger, 1933

Agrilus klugianus Obenberger, 1933

Agrilus kormilevi Bily, 1975

Agrilus laetabilis Kerremans, 1897

Agrilus laetitius Kerremans, 1897

Agrilus lamarcki Obenberger, 1933

Agrilus languens Kerremans, 1903

Agrilus languidus Chevrolat, 1838

Agrilus laomedon Obenberger, 1932

Agrilus laudabilis Kerremans, 1897

Agrilus lautus Kerremans, 1899

Agrilus lavillei Obenberger, 1932

Agrilus lavoisieri Obenberger, 1933

Agrilus leminus Obenberger, 1932

Agrilus lestageanus Obenberger, 1935

438 
Agrilus lineariformis Jendek, 2005

Agrilus lineellus Gory, 1841

Agrilus linnei Obenberger, 1933

Agrilus listreuon Obenberger, 1935

Agrilus loconte Curletti \& Brûlé, 2014b

Agrilus loesthus Obenberger, 1935

Agrilus longicornis Gory \& Laporte, 1837

Agrilus longiusculus Gory \& Laporte, 1837

Agrilus luanae Curletti, 2020a

Agrilus lucens Kerremans, 1897

Agrilus lucidicollis lucidicollis Gory, 1841

Agrilus lucidicollis rodens Obenberger, 1935

Agrilus luctuosus Kerremans, 1897

Agrilus luculentus Kerremans, 1900

Agrilus luderwaldti Obenberger, 1933

Agrilus lycaon Obenberger, 1933

Agrilus maculifrons Gory, 1841

Agrilus maderi Obenberger, 1932

Agrilus magnificientis Curletti \& Brulé, 2014

Agrilus manaosensis Obenberger, 1933

Agrilus mansuetus Thomson, 1879

Agrilus mariae Curletti, 2017

Agrilus martinsi Curletti \& Migliore, 2014b

Agrilus matho Obenberger, 1933

Agrilus medius Kerremans, 1900

Agrilus melancholicus Kerremans, 1897

Agrilus meleager meleager Obenberger, 1935

Agrilus meleager oeagrus Obenberger, 1935

Agrilus meracus Kerremans, 1897

Agrilus messorius Kerremans, 1903

Agrilus metuens Kerremans, 1903

Agrilus minimus Bellamy, 1998

Agrilus miron Obenberger, 1933

Agrilus modestus Gory \& Laporte, 1837

Agrilus monotonus Thomson, 1879

Agrilus moraguesi Curletti \& Brûlé, 2011

Agrilus mrazi Obenberger, 1933

Agrilus multispinosus (Klug, 1825)

Agrilus mundulus Obenberger, 1936

Agrilus munificus Kerremans, 1897

Agrilus najarae Curletti Ribeiro \& Migliore, 2016

Agrilus needhami Obenberger, 1933

Agrilus niger Gory \& Laporte, 1837

Agrilus nigerellus Thomson, 1879

Agrilus nigrofasciatus Gory \& Laporte, 1839

Agrilus nigror Curletti \& Brûlé, 2011

Agrilus nobilitatus Kerremans, 1899

Agrilus noctambulus Curletti \& Brûlé, 2011

Agrilus notabilis Kerremans, 1899

Agrilus novellus Curletti \& Brûlé, 2011

Agrilus oberthueri Kerremans, 1897 
Agrilus obscurellus Thomson, 1879

Agrilus obscuripennis Gory, 1841

Agrilus obsoletus Gory, 1841

Agrilus oceanicus Cobos, 1959

Agrilus octavius Obenberger, 1935

Agrilus oculifer Kerremans, 1899

Agrilus oliveirai Curletti, 2017

Agrilus omissus Kerremans, 1903

Agrilus omphax Obenberger, 1933

Agrilus opimus Kerremans, 1897

Agrilus orgetorix Obenberger, 1933

Agrilus ostentator Kerremans, 1900

Agrilus pabulator Obenberger, 1932

Agrilus pacificus Thomson, 1879

Agrilus palilogus Obenberger, 1933

Agrilus pallon Obenberger, 1933

Agrilus paolettae Curletti, Ribeiro \& Migliore, 2016

Agrilus paradelphus Obenberger, 1933

Agrilus paradoxus Boheman, 1858

Agrilus pattoni Obenberger, 1933

Agrilus paulista Curletti, 2016

Agrilus pauperculus Gory, 1841

Agrilus pedroi Obenberger, 1935

Agrilus perangustus Kerremans, 1897

Agrilus perplexus Burmeister, 1872

Agrilus pertenuis Kerremans, 1897

Agrilus pertristis Obenberger, 1933

Agrilus pes Curletti \& Brûlé, 2013b

Agrilus petronius Obenberger, 1933

Agrilus phanaeas Obenberger, 1933

Agrilus pictus Kerremans, 1897

Agrilus pinguis Kerremans, 1897

Agrilus pinhadaensis Obenberger, 1932

Agrilus pirilampo Curletti, Ribeiro \& Migliore, 2013

Agrilus pisinoe Gory, 1841

Agrilus placens Kerremans, 1897

Agrilus plicatus Kerremans, 1903

Agrilus postulator Kerremans, 1897

Agrilus praecipuus Kerremans, 1897

Agrilus praelucens Kerremans, 1897

Agrilus presli Obenberger, 1932

Agrilus preyssleri Obenberger, 1933

Agrilus prismaticus Kerremans, 1897

Agrilus productus Gory, 1841

Agrilus profugellus Thomson, 1879

Agrilus prolixus Kerremans, 1897

Agrilus pruinosus Chevrolat, 1838

Agrilus pseudosimilanus Obenberger, 1933

Agrilus pudens Kerremans, 1897

Agrilus puellus Gory \& Laporte, 1837

Agrilus puerilis Kerremans, 1897 
Agrilus pugionellus Thomson, 1879

Agrilus pulverosus Chevrolat, 1838

Agrilus puniceus Kerremans, 1897

Agrilus purus Kerremans, 1900

Agrilus pyropygus Thomson, 1879

Agrilus pyrosurus Gory \& Laporte, 1837

Agrilus quadriguttatellus Thomson, 1879

Agrilus quattuordecimsignatus Obenberger, 1935

Agrilus quietus Kerremans, 1897

Agrilus rafaeli Curletti, 2019b

Agrilus rarestriatus Curletti, Ribeiro \& Migliore, 2013

Agrilus rarus Kerremans, 1897

Agrilus redtenbacheri Obenberger, 1933

Agrilus reliquus Kerremans, 1903

Agrilus remus Obenberger 1935

Agrilus ribeiroi Curletti \& Migliore, 2014b

Agrilus rimosicollis Kerremans, 1897

Agrilus rodzjankoi Obenberger, 1933

Agrilus romulus Obenberger, 1935

Agrilus sacer sacer Kerremans, 1903

Agrilus sainthilairei Obenberger, 1933

Agrilus scrobicollis Thomson, 1879

Agrilus sculpturatus Kerremans, 1897

Agrilus sedyi Obenberger, 1933

Agrilus sekerai Obenberger, 1935

Agrilus sensitious Kerremans, 1897

Agrilus seriatus Curletti \& Brûlé, 2011

Agrilus servius Obenberger, 1935

Agrilus severini Obenberger, 1933

Agrilus sexflavoguttatellus Thomson, 1879

Agrilus sexguttatellus Thomson, 1879

Agrilus sexnotatus Gory \& Laporte, 1837

Agrilus sharpi Obenberger, 1933

Agrilus sicariellus Thomson, 1879

Agrilus similanus Obenberger, 1933

Agrilus similis Curletti \& Brûlé, 2013b

Agrilus simplicellus Thomson, 1879

Agrilus sincerus Kerremans, 1897

Agrilus siren Gory, 1841

Agrilus skrlandti Obenberger, 1933

Agrilus sorciellus Obenberger, 1932

Agrilus soricellus Thomson, 1879

Agrilus spiculum Curletti \& Brûlé, 2013b

Agrilus spinamajor Chevrolat, 1838

Agrilus spiniger Eschscholtz, 1822

Agrilus splendens Kerremans, 1897

Agrilus squalus Waterhouse, 1889

Agrilus srogli Obenberger, 1932

Agrilus stenachon Obenberger, 1933

Agrilus strandelinus Obenberger, 1936

Agrilus strandianus Obenberger, 1933 
Agrilus strigosus Kerremans, 1897

Agrilus strigulioides Obenberger, 1932

Agrilus suavis Kerremans, 1897

Agrilus subcongener Kerremans, 1899

Agrilus subdebilis Kerremans, 1899

Agrilus subinflatus Kerremans, 1899

Agrilus sulcatulus Chevrolat, 1835

Agrilus sulci Obenberger, 1932

Agrilus sumptuosissimus Obenberger, 1932

Agrilus suspiciosus Kerremans, 1897

Agrilus suturalineatus Thomson, 1879

Agrilus tabaci Kerremans, 1896

Agrilus taediosus Curletti, Ribeiro \& Migliore, 2013

Agrilus talthybius Obenberger, 1933

Agrilus templaris Curletti \& Brûlé, 2013b

Agrilus tenebricosus Kerremans, 1897

Agrilus tenuisculptus Obenberger, 1933

Agrilus terentius Obenberger, 1933

Agrilus tersus Kerremans, 1897

Agrilus thaumastus Obenberger, 1933

Agrilus thoracellus Gory \& Laporte, 1837

Agrilus tiberius Obenberger, 1933

Agrilus timidus Kerremans, 1897

Agrilus timorosus Curletti \& Migliore, 2014b

Agrilus tinantius Obenberger, 1960

Agrilus tragulus Obenberger, 1935

Agrilus transitorius Kerremans, 1897

Agrilus tridentatus Gory, 1841

Agrilus trimaculatus Gory, 1841

Agrilus triton Obenberger, 1935

Agrilus tubulinus Curletti, 2019b

Agrilus tubulus Kerremans, 1897

Agrilus tugio advocatus Obenberger, 1935

Agrilus tugio tugio Obenberger, 1935

Agrilus tullius Obenberger, 1933

Agrilus turgitus Kerremans, 1897

Agrilus unicolor Gory \& Laporte, 1837

Agrilus unicus Kerremans, 1897

Agrilus urbanus Kerremans, 1897

Agrilus utens Kerremans, 1897

Agrilus vagans Harold, 1869

Agrilus vaillanti Obenberger, 1935

Agrilus variabilis Kerremans, 1899

Agrilus variatus Kerremans, 1897

Agrilus venustulus Burmeister, 1872

Agrilus venustus Kerremans, 1897

Agrilus verax Kerremans, 1897

Agrilus versicolor Chevrolat, 1838

Agrilus verticis Curletti 2020a

Agrilus verutoides Obenberger, 1935

Agrilus verutus Kerremans, 1897

442 
Agrilus vescus Kerremans, 1897

Agrilus vespilio Kerremans, 1897

Agrilus villosulus Kerremans, 1897

Agrilus villus Curletti \& Brûlé, 2011

Agrilus violaceicollis Gory, 1841

Agrilus violacellus Thomson, 1879

Agrilus virescens Gory \& Laporte, 1837

Agrilus vitellius Obenberger, 1935

Agrilus vlachi Obenberger, 1933

Agrilus volucer Kerremans, 1897

Agrilus voluptuosus Kerremans, 1897

Agrilus votus Kerremans, 1897

Agrilus wasmanni wasmanni Obenberger, 1933

Agrilus xantholomus itaitubae Obenberger, 1932

Agrilus xantholomus xantholomus (Dalman, 1823)

Agrilus xiphion Obenberger, 1933

Agrilus yeti Curletti \& Migliore, 2014b

Agrilus zikani Obenberger, 1935

\section{Chile}

Agrilus aonikenk Cid-Arcos \& Pineda, 2019

Agrilus diaguita Moore, 1985

Agrilus sulcipennis Solier, 1849

Agrilus thoracicus Gory \& Laporte, 1837

Agrilus unipunctatus Gory \& Laporte, 1837

\section{Colombia}

Agrilus aculeipennis Chevrolat, 1838

Agrilus aleus Gory, 1841

Agrilus almenus Gory, 1841

Agrilus arnus Gory, 1841

Agrilus caquetai Curletti \& Dutto, 2017

Agrilus chrysostictus (Klug, 1825)

Agrilus claveri Kerremans, 1900

Agrilus colombianus Gory, 1841

Agrilus concupiens Obenberger, 1935

Agrilus conviva Obenberger, 1933

Agrilus crapulellus Thomson, 1879

Agrilus cupai Gory, 1841

Agrilus eupalamus Gory, 1841

Agrilus figuratus Curletti \& Brûlé, 2011

Agrilus florae Curletti \& Dutto, 2017

Agrilus frustrator Kerremans, 1900

Agrilus geminus Curletti \& Migliore, 2014b

Agrilus gracilipes Waterhouse, 1889

Agrilus hectori Curletti, 2005

Agrilus hilaris Waterhouse, 1889

Agrilus honorius Obenberger, 1933

Agrilus ineditus Chevrolat, 1838

Agrilus maculatus Gory \& Laporte, 1837

Agrilus pinedai Curletti, 2020a 
Agrilus piscis Gory, 1841

Agrilus pisinoe Gory, 1841

Agrilus scopiazon Obenberger, 1933

Agrilus siren Gory, 1841

Agrilus trispinosus Gory, 1841

\section{Ecuador}

Agrilus aequatoris Obenberger, 1917

Agrilus aurifrons Kerremans, 1897

Agrilus delsinnei Curletti, 2015a

Agrilus narcissus Obenberger, 1917

Agrilus pisander Obenberger, 1933

Agrilus quito Curletti, 2015a

Agrilus tandapianus Curletti, 2015c

Agrilus touroultinus Curletti, 2015a

Agrilus yanzatzanus Curletti, 2015a

\section{Guyana}

Agrilus centurial Jendek, 2001

Agrilus ceruleodepilis Thomson, 1879

Agrilus conformis Gory, 1841

Agrilus irrequietus Thomson, 1879

Agrilus lacordairei Gory \& Laporte, 1837

Agrilus setipes Chevrolat, 1838

\section{Guyane Française}

Agrilus abrasus Curletti \& Brûlé, 2011

Agrilus adamsoni Fisher, 1944

Agrilus agnitus Curletti \& Brûlé, 2013a

Agrilus alienus Curletti \& Brûlé, 2015b

Agrilus amoenus Curletti \& Brûlé, 2014a

Agrilus analis Kerremans, 1896

Agrilus antelucanus Curletti \& Brûlé, 2017

Agrilus anthracites Curletti \& Brûlé, 2017

Agrilus antoinei Curletti \& Brûlé, 2013a

Agrilus araneipes Obenberger, 1932

Agrilus atrox Curletti \& Brûlé, 2017

Agrilus auratolineatus Curletti \& Brûlé, 2011

Agrilus auroscutatus Curletti \& Brûlé, 2011

Agrilus avarus Curletti \& Brûlé, in press

Agrilus bacchanalis Curletti \& Brûlé, in press

Agrilus balatanus Curletti \& Brûlé, 2015a

Agrilus bifidus Curletti \& Brûlé, 2014a

Agrilus bikini Curletti \& Brûlé, 2013b

Agrilus bison Curletti \& Brûlé, 2015b

Agrilus cacuminatus Curletti \& Brûlé, 2015b

Agrilus calvoventre Curletti \& Brulé, 2013b

Agrilus camusi Curletti \& Brûlé, 2011

Agrilus capucinus Curletti \& Brûlé, 2014a

Agrilus centurial Jendek, 2001

Agrilus cerdai Curletti \& Brûlé, 2011 
Agrilus cibarius Fisher, 1944

Agrilus cimmerius Curletti \& Brûlé, 2015b

Agrilus coeruleodepilis Thomson, 1879

Agrilus colleti Curletti \& Brûlé, 2011

Agrilus collis Curletti \& Brûlé, 2013b

Agrilus consimilis Waterhouse, 1899

Agrilus consuetus Curletti \& Brûlé, 2015a

Agrilus decarinatus Curletti \& Brûlé, 2013b

Agrilus depilatus Curletti \& Brûlé, 2011

Agrilus dewynteri Curletti \& Brûlé, 2015b

Agrilus diromai Curletti \& Brûlé, 2011

Agrilus dirus Kerremans, 1897

Agrilus dives Kerremans, 1897

Agrilus durantoni Curletti \& Brûlé, 2011

Agrilus eburneus Curletti \& Brûlé, 2013b

Agrilus elfus Curletti \& Brûlé, 2017

Agrilus emicyaneus Curletti \& Brûlé, 2016

Agrilus empictus Curletti \& Brûlé, 2017

Agrilus emsignatus Curletti \& Brûlé, 2017

Agrilus epaulus Obenberger, 1932

Agrilus esasignatus Curletti \& Brûlé, 2016

Agrilus excelsus Curletti \& Brûlé, 2015b

Agrilus falsus Curletti \& Brûlé, 2013a

Agrilus fanericus Curletti \& Brûlé, 2013b

Agrilus faurei Curletti \& Brûlé, 2011

Agrilus fei Curletti \& Brûlé, 2017

Agrilus fernandezi Curletti \& Brûlé, 2013b

Agrilus figuratus Curletti \& Brûlé, 2011

Agrilus florens Curletti \& Brûlé, in press

Agrilus forceps Curletti \& Brûlé, 2014a

Agrilus forficulus Curletti \& Brûlé, 2013a

Agrilus fourgassiei Curletti \& Brûlé, 2013a

Agrilus fragmentatus Curletti \& Brûlé, 2011

Agrilus furcatipennis (Gory \& Laporte, 1837)

Agrilus giuglarisi Curletti \& Brûlé, 2011

Agrilus glaber Curletti \& Brûlé, 2013a

Agrilus gloriae Curletti \& Brûlé, 2018

Agrilus goryellus Thomson, 1879

Agrilus goryi Saunders, 1870

Agrilus inauratus Curletti \& Brûlé, 2014a

Agrilus incredulus Curletti, 2005

Agrilus intermixtus Curletti \& Brûlé, in press

Agrilus irrequietus Thomson, 1879

Agrilus ittericus Curletti \& Brulé, in press

Agrilus jenningsi Fisher, 1938

Agrilus kapiriensis Curletti \& Brûlé, 2013b

Agrilus kawi Curletti \& Brûlé, 2017

Agrilus lacordairei Gory \& Laporte, 1837

Agrilus lacrimans Curletti \& Brûlé, 2013b

Agrilus lamatus Curletti \& Brûlé, 2011

Agrilus laminatus Curletti \& Brûlé, 2011 
Agrilus lavalettei Curletti \& Brulé, 2014b

Agrilus lignicida Curletti \& Brûlé, 2011

Agrilus limesignatus, Curletti \& Brûlé, 2011

Agrilus lindae Curletti \& Brulé, 2017

Agrilus loconte Curletti \& Brûlé, 2014b

Agrilus loesthus Obenberger, 1935

Agrilus longelineatus Curletti \& Brûlé, 2011

Agrilus lopus Obenberger, 1935

Agrilus macilentus Curletti \& Brûlé, 2013b

Agrilus maeror Curletti \& Brûlé, 2011

Agrilus magnificientis Curletti \& Brulé, 2014

Agrilus mareki Curletti \& Brûlé, 2015a

Agrilus matitinus Curletti \& Brûlé, 2014b

Agrilus matourinus Curletti \& Brûlé, 2014b

Agrilus meloe Curletti \& Brûlé, 2011

Agrilus midas Curletti \& Brûlé, 2014a

Agrilus minissimus Curletti \& Brûlé 2011

Agrilus miserandus Curletti \& Brûlé, 2013a

Agrilus miserrimus Curletti \& Brûlé, 2016

Agrilus moraguesi Curletti \& Brûlé, 2011

Agrilus morvanae Curletti \& Brûlé, 2011

Agrilus murmillo Curletti \& Brûlé, 2013b

Agrilus musculosus Curletti \& Brûlé, 2014b

Agrilus mysticus Curletti \& Brûlé, 2013b

Agrilus nigrobrunneus Curletti \& Brûlé, 2017

Agrilus nigror Curletti \& Brûlé, 2011

Agrilus nobilitatus Kerremans, 1899

Agrilus noctambulus Curletti \& Brûlé, 2011

Agrilus novelliformis Curletti \& Brûlé, 2013a

Agrilus novellus Curletti \& Brûlé, 2011

Agrilus nudoventre Curletti \& Brûlé, 2014b

Agrilus oberthueri Kerremans, 1897

Agrilus oberthuroides Curletti \& Brûlé, 2016

Agrilus oinoicus Curletti \& Brûlé, 2015a

Agrilus orgiasticus Curletti \& Brûlé, 2013b

Agrilus partimcinctus Curletti \& Brûlé, 2011

Agrilus patawaensis Curletti \& Brûlé, 2011

Agrilus perditus Curletti \& Brûlé, 2011

Agrilus pes Curletti \& Brûlé, 2013b

Agrilus phallicus Curletti \& Brûlé, 2011

Agrilus piscis Gory, 1841

Agrilus plecotus Curletti \& Brûlé, 2011

Agrilus poirieri Curletti \& Brûlé, 2011

Agrilus productus Gory, 1841

Agrilus pudendus Curletti \& Brûlé, 2014a

Agrilus reginensis Curletti \& Brulé, 2014b

Agrilus rosanti Curletti \& Brûlé, 2011

Agrilus rubrofuscus Curletti \& Brûlé, 2013b

Agrilus rubronigromaculatus Curletti \& Brûlé, 2015b

Agrilus serialis Curletti \& Brûlé, 2013b

Agrilus seriatus Curletti \& Brûlé, 2011 
Agrilus setipes Chevrolat, 1838

Agrilus sexnotatus Gory \& Laporte, 1837

Agrilus sigillum Curletti \& Brûlé, 2013b

Agrilus silvanoi Curletti \& Brûlé, 2017

Agrilus similis Curletti \& Brûlé, 2013b

Agrilus similiter Curletti \& Brûlé, 2014a

Agrilus simulator Curletti \& Brûlé, 2011

Agrilus slami Curletti \& Brûlé, 2015b

Agrilus spiculatum Curletti \& Brulé, 2014b

Agrilus subdebilis Kerremans, 1899

Agrilus templaris Curletti \& Brûlé, 2013b

Agrilus torvus Curletti \& Brûlé, 2011

Agrilus touroulti Curletti \& Brûlé, 2011

Agrilus trames Curletti \& Brûlé, 2013b

Agrilus tristani Curletti \& Brûlé, 2011

Agrilus velox Curletti \& Brûlé, 2011

Agrilus vergineus Curletti \& Brûlé, 2014b

Agrilus vermiculus, Curletti \& Brûlé, in press

Agrilus vespillo Curletti \& Brûlé, 2011

Agrilus villus Curletti \& Brûlé, 2011

Agrilus virginiae Curletti \& Brûlé, 2013b

\section{Paraguay}

Agrilus albomaculifer Saunders, 1870

Agrilus amazonicus Kerremans, 1897

Agrilus antali Obenberger, 1933

Agrilus aurocephalus Gory, 1841

Agrilus bruchi Kerremans, 1903

Agrilus chrysostictus (Klug, 1825)

Agrilus cuvieri Obenberger, 1933

Agrilus desideratus Kerremans, 1897

Agrilus dilaticornis dilaticornis Kerremans, 1897

Agrilus faciatus Kerremans, 1903

Agrilus frigidus Gory, 1841

Agrilus goyaz Bellamy, 1998

Agrilus gratiosulus (Obenberger, 1932)

Agrilus guarani Obenberger, 1933

Agrilus hinnulus Obenberger, 1933

Agrilus leminus Obenberger, 1932

Agrilus neocles Obenberger, 1932

Agrilus parasimilanus Obenberger, 1933

Agrilus putzeysi Obenberger, 1933

Agrilus pylnovi Obenberger, 1933

Agrilus ruzzieri Curletti, 2016

Agrilus sceptuchus Obenberger, 1933

Agrilus schreineri Obenberger, 1933

\section{Peru}

Agrilus adelungi Obenberger, 1933

Agrilus alatus Curletti \& Pineda, 2019

Agrilus atahualpa Obenberger, 1935 
Agrilus augustus Obenberger, 1933

Agrilus bikini Curletti \& Brûlé, 2013b

Agrilus cordillerae Kirsch, 1873

Agrilus dilleri Curletti, 2020b

Agrilus duplicatus Kerremans, 1896

Agrilus figuratus Curletti \& Brûlé, 2011

Agrilus floreni Curletti, 2020b

Agrilus geminus Curletti \& Migliore, 2014b

Agrilus globulus preandinus Curletti, 2020b

Agrilus hilaris Waterhouse, 1889

Agrilus hornburgianus Curletti, 2020b

Agrilus huascar Obenberger, 1932

Agrilus icanus Curletti, 2020a

Agrilus iquitosanus Curletti, 2019a

Agrilus loconte Curletti \& Brûlé, 2014

Agrilus maculatus Gory \& Laporte, 1837

Agrilus morsoventre Curletti, 2020b

Agrilus nudoventre Curletti \& Brûlé, 2014b

Agrilus panguanae Curletti, 2020b

Agrilus perfectus Curletti, 2020b

Agrilus piuraensis Juarez \& Gonzales, 2018

Agrilus plurimus Curletti, 2020b

Agrilus pustulatus Curletti, 2020b

Agrilus rafaeli Curletti, 2019b

Agrilus semirufus Curletti, 2019a

Agrilus setipes Chevrolat, 1838

Agrilus similanus Obenberger, 1933

Agrilus similis Curletti \& Brûlé, 2013b

Agrilus wachteli Curletti, 2020b

Agrilus westerduijni Curletti, 2019b

Agrilus xantholomus xantholomus (Dalman, 1823)

\section{Trinidad \& Tobago}

Agrilus adamsoni Fisher, 1944

Agrilus animatus Fisher, 1944

Agrilus callani Fisher, 1943

Agrilus captivus Fisher, 1944

Agrilus cibarius Fisher, 1944

Agrilus consumptoris Fisher, 1944

Agrilus esculentus Fisher, 1944

Agrilus hanoti Curletti, 2019a

Agrilus hostia Fisher, 1944

Agrilus picinus Fisher, 1943

Agrilus predae Fisher, 1943

Agrilus trinidadensis Fisher, 1943

Agrilus victima Fisher, 1944

\section{Uruguay}

Agrilus baeri Obenberger, 1933

Agrilus bellicus Kerremans, 1903

Agrilus canelonius Obenberger, 1933

448 
Agrilus chacoensis Obenberger, 1935

Agrilus confossicollis Kerremans, 1892

Agrilus frigidinus Obenberger, 1932

Agrilus obscuripennis Gory, 1841

Agrilus sacer tremolerasi Obenberger, 1933

Agrilus telaemenes Obenberger, 1933

Agrilus unipunctatus Gory \& Laporte, 1837

Agrilus uruguayanus Obenberger, 1933

\section{Venezuela}

Agrilus arnoldi Curletti, 2020b

Agrilus bordoniellus Curletti, 2020b

Agrilus cabellanus Kerremans, 1895

Agrilus caracanus Obenberger, 1933

Agrilus caucus Curletti, 2020b

Agrilus coniectum Curletti, 2020b

Agrilus crapulellus Thomson, 1879

Agrilus episcopalis Curletti, 2020b

Agrilus erbeniellus Obenberger, 1935

Agrilus homocletes Obenberger, 1933

Agrilus improbabilis Curletti, 2020b

Agrilus lacordairei Gory \& Laporte, 1837

Agrilus mendacium Curletti, 2020b

Agrilus miron Obenberger, 1933

Agrilus ningitus Curletti, 2020b

Agrilus petarensis Kerremans, 1895

Agrilus probabilis Curletti, 2020b

Agrilus probus Kerremans, 1897

Agrilus prudens Kerremans, 1897

Agrilus pylnovi Obenberger, 1933

Agrilus quadrispilotus Obenberger, 1935

Agrilus simoni Kerremans, 1895

Agrilus siren Gory, 1841

Agrilus templaris Curletti \& Brûlé, 2013b

Agrilus wichmanniellus Obenberger, 1960

\section{Literature Cited}

Bellamy, C.L. (2008) A World Catalogue and Bibliography of the Jewel Beetles (Coleoptera: Buprestoidea). Volume 4: Agrilinae: Agrilina through Trachyini. Sofia-Moscow, Pensoft Publishers. pp. 1932-2684.

Cid-Arcos, M. and Pineda, C. (2019) Notas sobre Agrilus Curtis (Coleoptera: Buprestidae: Agrilinae), con descripción de una nueva especie para Chile. Revista Chilena de Entomología, 45(3):347-352.

Curletti, G. (2013) Un caso d'omonimia nel genere Agrilus Curtis, 1825. Rivista piemontese di Storia naturale, 34: 242.

Curletti, G. (2015a) Four new species of the genus Agrilus Curtis, 1825 from Ecuador (Coleoptera, Buprestidae). Zootaxa, 3985(4): 575-582.

Curletti, G. (2015b) Three new species of Agrilini (Coleoptera Buprestidae) from Argentina and Bolivia. Giornale italiano di Entomologi, 14(60): 25-30.

Curletti, G. (2015c) Two new species of the genus Agrilus Curtis, 1825 from Ecuador and Nicaragua (Coleoptera, Buprestidae). Rivista piemontese di Storia naturale, 36: 219-223. 
Curletti, G. (2016) New Neotropical Agrilus species. Rivista piemontese di Storia Naturale, 37: 211-223.

Curletti, G. (2018a) Appunti sugli Agrilus Curtis, 1825 dell'Argentina (Coleoptera, Buprestidae). Rivista piemontese di Storia naturale, 39: 317-346.

Curletti, G. (2018b) Mata Atlantica e Mata Amazonica. Comparazioni sullo stato di conoscenza degli Agrilini tra le due regioni. Conferenza presso l'Instituto de Pesquisas da Amazônia, Manaus.

Curletti, G. (2019a) Nuove specie tropicali del genere Agrilus Curtis, 1825 (Coleoptera, Buprestidae). Bollettino del Museo Regionale di Scienze Naturali di Torino, 35(1-2): 33-58.

Curletti, G. (2019b) The Agrilini of the Instituto Nacional de Pesquisas da Amazônia (INPA), Manaus, Brazil, with faunistic considerations on the Brazilian Mata Amazônica (Coleoptera, Buprestidae). Giornale Italiano di Entomologia, 15(64): 413-426.

Curletti, G. (2020a) Nuevas adiciones al conocimiento taxonómico del género Agrilus Curtis (Coleoptera: Buprestidae: Agrilinae) de la Región Neotropical. Revista Chilena de Entomología, 46(2): 243-254.

Curletti, G. (2020b) Nuovi taxa di Agrilini da Perù e Venezuela (Coleoptera, Buprestidae). Giornale Italiano di Entomologia, 15(64): 657-682.

Curletti, G. (2020c) Tre nuove specie di Agrilini della foresta atlantica brasiliana (Coleoptera, Buprestidae). Rivista piemontese di Storia naturale, 41: 149-152.

Curletti, G. and Brûlé, S. (2011) Agrilus, Agriloides et Autarcontes de Guyane. Magellanes ed. Ex Natura, 2: 1-86.

Curletti, G. and Brûlé, S. (2013a) De nouvelles espèces d'Agrilus pour la Guyane Française issues de la collection J. Marek (Coleoptera, Buprestidae, Agrilinae). Magellanes ed. Ex Natura, 5: 45-63.

Curletti, G. and Brûlé, S. (2013b) Deuxième contribution à la connaissance des Agrilini de Guyane (Coleoptera, Buprestidae, Agrilinae). Magellanes ed. Ex Natura, 5: 1-44.

Curletti, G. and Brûlé, S. (2014a) Quatrième contribution à la connaissance des Agrilini de Guyane. Giornale Italiano di Entomologia, 13(59): 613-626.

Curletti, G. and Brûlé, S. (2014b) Sixième contribution à la connaissance des Agrilini de Guyane. ACOREP, France, Coléoptères de Guyane, VIII: 40-51.

Curletti, G. and Brûlé, S. (2015a) Cinquième contribution à la connaissance des Agrilini de Guyane (Coleoptera, Buprestidae, Agrilinae). Giornale Italiano di Entomologia, 14(60): 71-78.

Curletti, G. and Brûlé, S. (2015b) Septième contribution à la connaissance des Agrilini de Guyane (Coleoptera, Buprestidae). ACOREP France, Coléoptères de Guyane, IX: 43-60.

Curletti, G. and Brûlé, S. (2016) Huitième contribution à la connaissance des Agrilini de Guyane (Coleoptera, Buprestidae). ACOREP-France, Coléoptères de Guyane, X: 52-57.

Curletti, G. and Brûlé, S. (2017) Neuvième contribution à la connaissance des Agrilini de Guyane(Coleoptera, Buprestidae). ACOREP France, Coléoptères de Guyane, XI: 14-25.

Curletti, G. and Brûlé, S. (2018) Agrilus gloriae n. sp. de Guyane (Coleoptera, Buprestidae). Dixième contribution à la connaissance des Agrilini de Guyane. ACOREP France, Coléoptères de Guyane, XII: 10-11.

Curletti, G. and Dutto, A. (2017) Notes on the Agrilo-fauna of the Colombian Amazon (Coleoptera, Buprestidae). Zootaxa, 4243(2): 373-376.

Curletti, G. and Migliore, L. (2013) A new species of Agrilus Curtis, 1825 from the Natural History Museum of Porto Alegre, Brazil. Giornale Italiano di Entomologia, 13(58): 351-354.

Curletti, G. and Migliore, L. (2014a) A new species of Agrilus Curtis, 1825 from Brazil (Coleoptera, Buprestidae). Biodiversity Journal, 5(2): 197-200.

Curletti, G. and Migliore, L. (2014b) O gênero Agrilus Curtis, 1829 nas coleções do Museu de Zoologia da Universidade de São Paulo. Papéis Avulsos de Zoologia, 54(8): 81-106.

Curletti, G. and Pineda, C. (2018) Nuevas especies del género Agrilus Curtis (Coleoptera: Buprestidae: Agrilinae) del Estado de Paraná, Brasil. Revista Chilena de Entomología, 44(1): 5-8. 
Curletti, G. and Pineda, C. (2019) Nuevas especies de la tribu Agrilini Laporte (Coleoptera: Buprestidae: Agrilinae) de América del Sur. Revista Chilena de Entomología, 45(2): 195-199.

Curletti, G., Ribeiro, P.S. and Migliore, L. (2013) Preliminary contribution to the knowledge of Coleoptera Buprestidae from Atlantic rainforest regions of Rio Doce and Itacolomi State Parks of Minas Gerais, Brazil. The genera Agrilus Curtis, 1825, Autarcontes Waterhouse, 1887, and Geralius Harold, 1869. Biodiversity Journal, 4(4): 443-450.

Curletti, G., Ribeiro, P.S. and Migliore, L. (2016) Further three new species of Agrilus Curtis, 1825 from Brazil (Coleoptera: Buprestidae). Onychium, 12: 129-134.

Hespenheide, H.A. and Chaboo, C.S. (2015) Beetles (Coleoptera) of Peru: A Survey of the Families. Buprestidae. Journal of the Kansas Entomological Society, 88(2): 211-214.

Jendek, J. (2012) Corrections to Agrilus related species-group names in the world catalogue of Bellamy and new substitute names for Agrilus species-group homonyms. ZooKeys, 249: 61-76.

Juárez, G. and González, U. (2017) Una especie nueva del género Agrilus Curtis, 1825 (Coleoptera, Buprestidae) de Perú. Boletín de la Sociedad Entomológica Aragonesa (S.E.A.), 60: 277-279. 